\title{
Hirschsprung's disease associated with congenital heart malformation, broad big toes, and ulnar polydactyly in sibs: a case for fetoscopy
}

\author{
K. M. LAURENCE,* R. PROSSER, $†$ I. ROCKER, $\ddagger$ J. F. PEARSON, ${ }^{* *}$ and \\ C. RICHARDS $\ddagger$
}

Summary. Successful fetoscopy using a $9 \mathrm{~mm}$ laparoscope was carried out on an 18-week pregnancy of a healthy woman who had had two previous male infants with bilateral double big toes, bilateral ulnar supernumerary digits associated with short segment Hirschsprung's disease, and ventricular septal defect of the heart, a syndrome apparently not previously described.

The fetus was found to be normal, but an amniotic membrane defect was detected, which accounted for amniotic fluid leakage from 24 weeks up to delivery by caesarean section of a normal male infant at 35 weeks.

The possible genetic basis and recurrence risk, put at probably one in four for this syndrome, is discussed. The indications for fetoscopy are set out and a fundal approach at laparotomy after placental localization is recommended for the procedure.

Hirschsprung's disease is generally regarded to be one of the conditions where genetic factors, probably polygenic in nature, are important in the aetiology (Bodian and Carter, 1963). Various associated malformations have been recorded in about $10 \%$ of cases. Only Down's syndrome and possibly pyloric stenosis seems to occur rather more frequently than might be expected by chance (Passarge, 1967). A hitherto undescribed association with almost identical cardiac and skeletal abnormalities in two male sibs (Laurence et al, 1974), which led to the successful use of fetoscopy in a subsequent pregnancy, is the subject of this report.

\section{Case reports}

Case 1 (III.5) was born on 8 December 1966. The pregnancy though 42 weeks long was uneventful, and the delivery, after spontaneous onset of labour, was normal. The male infant, birth weight $3830 \mathrm{~g}$, was noted to have

Received 13 December 1974.

* Department of Child Health, Welsh National School of Medicine, Heath Park, Cardiff.

t Department of Child Health, Royal Gwent Hospital, Newport.

$\mp$ Department of Obstetrics, Royal Gwent Hospital, Newport.

** Department of Obstetrics, Welsh National School of Medicine, Heath Park, Cardiff. bilateral supernumerary digits on the ulnar side of the hand with a fleshy connexion only. These were removed by ties with silk, leaving small stumps (Fig. 1). $\mathrm{He}$ also had a very distinctive broad double big toe on each foot (Fig. 2 and 3), a degree of phimosis, and a husky cry. At the end of the first day he had not passed meconium; rectal examination showed the anus to be tight and the rectum to be empty, while the abdomen was distended with several easily palpable sausage-shaped masses. The infant was dyspnoeic but there was no clinical cardiomegaly, abnormal heart sound, or cyanosis. On the second day he was transferred to a surgical unit where a laparotomy was performed which showed the rectum and lower portion of the sigmoid colon to be collapsed and the rest of the large bowel to be dilated. A mucosal biopsy was taken which contained large bundles of nerve fibres in the sub-mucosa and between the muscle layers, with the absence of ganglia, suggesting that this was a case of Hirschsprung's disease of the short segment variety. A right transverse colostomy was performed. The postoperative course was somewhat stormy and at the end of the first week a grade 2 systolic murmur was heard for the first time, together with an increased pulmonary second sound. Cardiac enlargement was noted and an ECG confirmed right ventricular hypertrophy. Subsequent investigation, including catheterization and cine angiography, showed the cardiac lesion to be a partial mitral stenosis with pulmonary hypertension and 


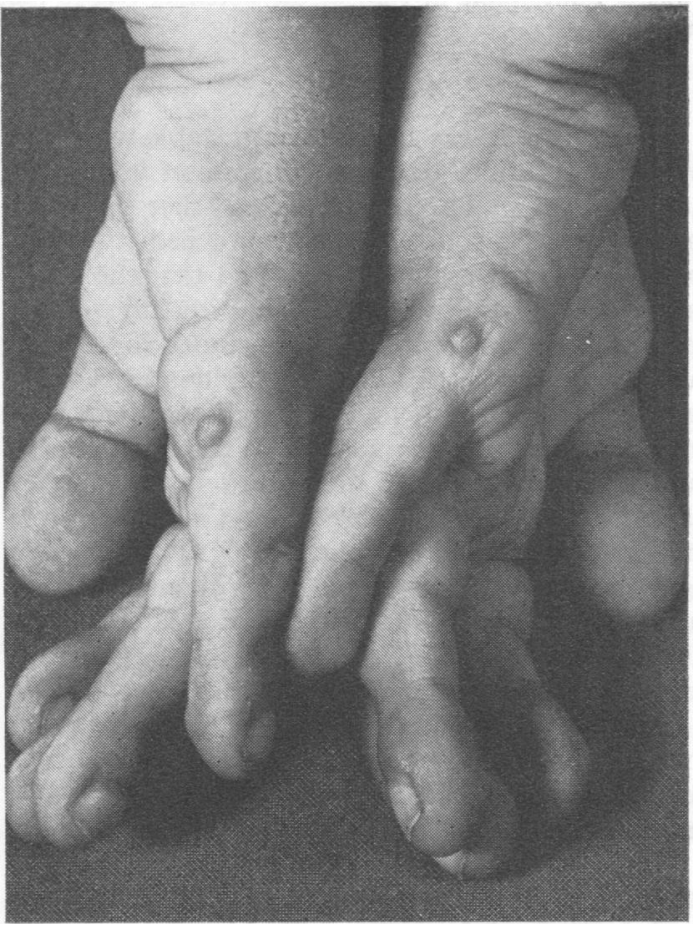

Fig. 1. Case 1. The ulnar aspect of the hands showing the stumps where the supernumerary digits had been removed at birth.

a ventricular septal defect. A biochemical profile, urinary amino-acid scan, and tests for mucopolysaccharides were all negative. Chromosome analysis, carried out after peripheral blood culture, showed him to have a normal male chromosome constitution $(46, \mathrm{XY})$.

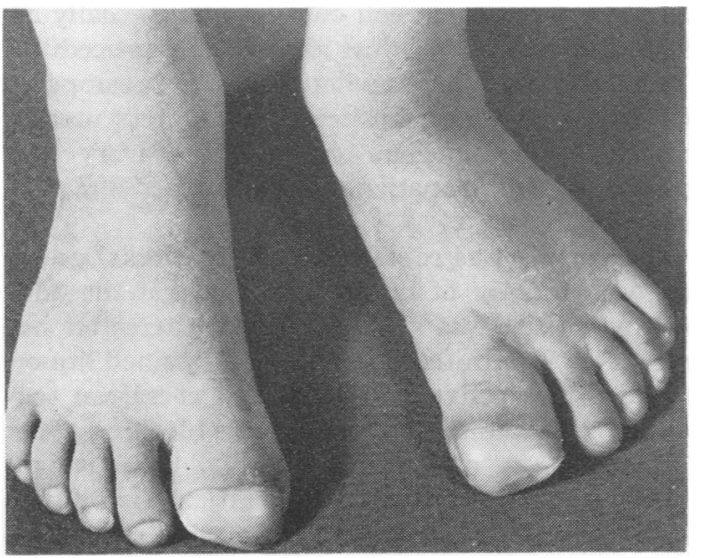

FIg. 2. Case 1. The feet at 5 years showing the broad big toes with a depression down each nail, suggesting syndactyly.
At the age of $2 \frac{1}{2}$ years a successful Swenson's rectosigmoidectomy and pull-through operation was carried out. One month later the colostomy was closed. He has had frequent admissions to hospital subsequently for severe respiratory distress with bronchospasm and has at times been in cardiac failure, but these episodes have lessened as he grew older. At the age of $7 \frac{1}{2}$ years, he is $116 \mathrm{~cm}$ high and weighs $2320 \mathrm{~g}$, is of normal intellect, and is relatively fit with a reasonable exercise tolerance, though he had quite marked finger clubbing (Fig. 1). He has moderate abdominal distension and reasonable bowel action. He is waiting for corrective cardiac surgery to be carried out in due course.

Case 2 (III.6) was born on 10 July 1968 after an uneventful 38-week pregnancy and a normal delivery; birth weight was $3410 \mathrm{~g}$. He was noted to have exactly the same extra digit on the ulnar side of both hands and the double big toe on each foot, the same phimosis, and husky cry as his elder brother. Meconium had not been passed on the first day and his rectum was empty, though on the second day he did pass some and appeared to be feeding well. Cardiac enlargement became apparent, there was a soft systolic murmur; chest radiology showed cardiac enlargement, and an ECG demonstrated right axis deviation with right ventricular hypertrophy. On the third day he developed obvious cardiac failure with tachycardia, liver enlargement, and peripheral oedema. A definite grade 2 systolic murmur was now audible together with an accentuated pulmonary second sound. He was digitalized and given diuretics. In the meanwhile, the abdomen became distended with evidence of intestinal obstruction and in spite of his poor condition a laparotomy was carried out on the 11 th day and he was given a transverse colostomy. Postoperatively, the colostomy never functioned, he developed peritonitis and rapid progressive cardiac failure and died 3 days later, aged 2 weeks.

Necropsy revealed that apart from peritonitis due to a

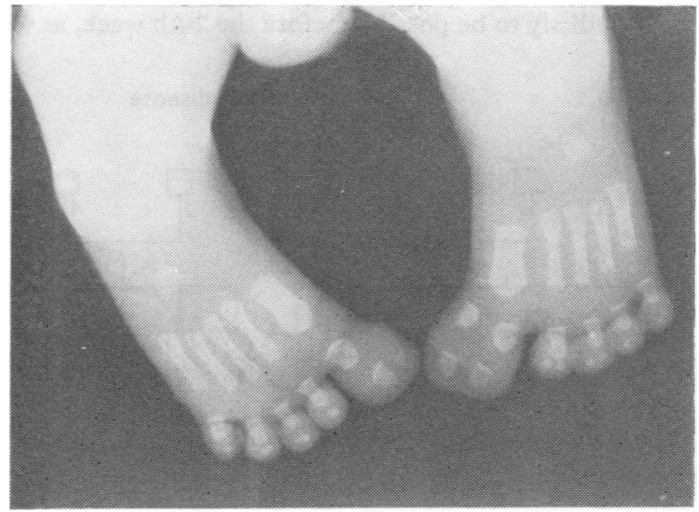

FIG. 3. Case 1. Radiograph of the feet showing bilateral polydactyly and syndactyly of the big toes. 
perforated caecum, he had short segment Hirschsprung's disease with hypertrophic nerve bundles, and absent ganglion cells in the rectal mucosa. The enlarged heart showed right atrial and ventricular hypertrophy and dilatation, tricuspid stenosis, and moderate mitral stenosis. The ductus arteriosus was widely patent and there was a moderate degree of preductal coarctation of the aorta. No ventricular septal defect was present.

\section{Family history}

The father (II.7) a pipe fitter, and the mother (II.8) a part-time hairdresser, both born in 1948, were healthy and free from any obvious skeletal abnormality. There was no family history of any abnormality, apart from a 12-week miscarriage (III.8) in the mother's only sister, two 12-week miscarriages (III.2 and III.3) in the father's only sister, and also his mother's two miscarriages (II.1 and II.2). There is no consanguinity (Fig. 4).

A year after the birth of the second son the parents sought genetic advice. If the case was regarded simply as a case of Hirschsprung's disease, it was felt that, with a risk of recurrence for boys of 1 in 20 after one child with short segment Hirschsprung's disease (Bodian and Carter, 1963), this should probably be doubled to about 1 in 10 after a second affected boy. It was considered more likely, however, that the patients had a new syndrome, of which aganglionic megacolon was only a part, and in that case the recurrence risk was likely to be close to 1 in 4 . On the second assumption it was also likely that another affected fetus would have similar skeletal abnormalities and also probably cardiac malformations.

Two years later the couple wished to proceed with a further pregnancy, but in view of the high risk of recurrence they asked for at least some assurance that the fetus did not have the same abnormalities as the previous sibs. In view of the absence of any chromosome or biochemical abnormality in the one sib at least, mid-trimester amniocentesis followed by cytogenetics or biochemistry, would not have been helpful and the only prospect of prenatal diagnosis lay in detecting the external skeletal malformations. Radiological detection of the double big toe, though distinctive at term, was thought to be difficult in utero, most unlikely to be possible before the 20 th week, as well

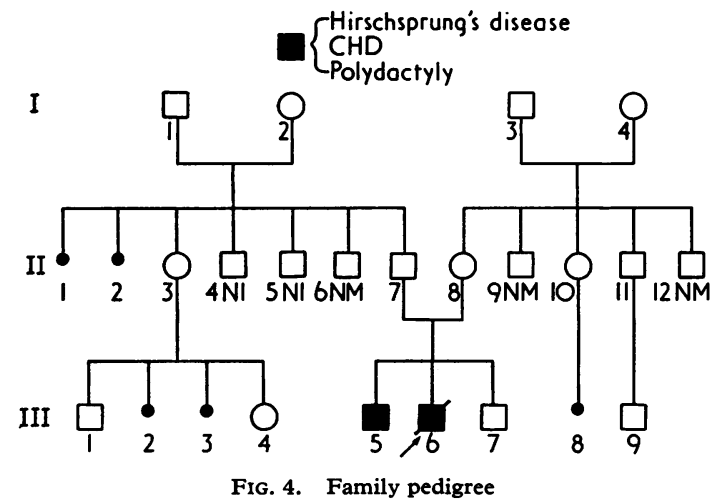

as being undesirable in view of the potential risk to the fetus.

\section{Fetoscopy}

As the mother was an intelligent, stable woman it was agreed to offer fetoscopy in spite of its experimental nature. The parents agreed that the pregnancy would only be allowed to proceed if normal hands and feet were visualized, and that the pregnancy would be terminated if abnormalities of the limbs were seen or could not be excluded for technical or other reasons. Thirteen previous fetoscopies had been carried out, followed by immediate hysterotomy or hysterectomy, before it was attempted in this case. In all women the duration of pregnancy was between 14 and 20 weeks, and in none was there any postoperative complication. A variety of rigid and flexible endoscopes with diameters ranging from 2 to $16 \mathrm{~mm}$ were tried. Finally a Richard Wolf $9 \mathrm{~mm}$ rigid end-viewing laparoscope, being the one which provided a wide field of vision, a good depth of focus, and good illumination was chosen. This instrument with a trocar and cannula including a non-return valve, reduced liquor loss to a minimum.

Since ultrasound had established an anterior placenta, a midline posterior fundal approach was used through a left para-umbilical laparotomy at 18 weeks' gestation. A $1.27 \mathrm{~cm}$ uterine incision was made into the gap of a wide horizontal mattress suture. The catgut suture acted as a stay while the incision was deepened almost to the depth of the? membranes. After perforation of the remaining muscle and membrane with the trocar, fetoscopy was undertaken with the $9 \mathrm{~mm}$ Wolf laparoscope. The three observers present agreed that both hands and the right foot were normal. Eight $\mathrm{ml}$ of liquor was taken for amnion cell culture, and virtually no additional liquor was lost during the procedure. The uterine incision was sutured and a postoperative isoxsuprine infusion helped to depress uterine activity. Immediate postoperative recovery was uneventful, and the patient was discharged after one week's bedrest.

The patient was re-admitted at 26 weeks' gestation with leakage of liquor, and again at the 28th week when she was kept in hospital thereafter because of an intermittent loss of blood-stained liquor. Delivery by elective lower segment caesarean section was precipitated by more severe bleeding at the 35th week. A male infant (III.7), birth weight $2880 \mathrm{~g}$, was delivered. He seemed externally normal and subsequent investigation has shown no evidence of either Hirschsprung's disease or cardiac malformation. 


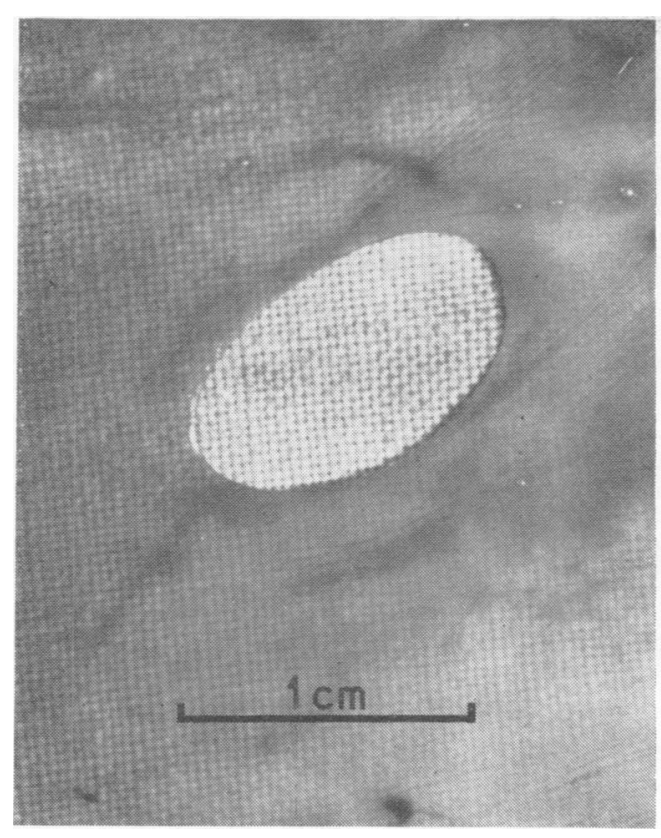

Fig. 5. The ovoid defect in the membranes, in about the position where the laparoscope was inserted into the uterus.

At delivery the fetoscopy scar was examined and no evidence of dehiscence of the uterine wall was seen. The subjacent membranes, however, contained a $1.2 \mathrm{~cm}$ diameter ovoid defect (Fig. 5), the edge of which was thickened because of liquefaction degeneration between amnion and chorion, suggesting an old lesion.

The father has subsequently had a vasectomy.

\section{Discussion}

In most of the larger reported series including those of Wylie (1957), Bodian and Carter (1963), Hofmann and Rehbein (1966), and Fraser and Wilkinson (1967), Hirschsprung's disease seems to be associated with other malformation in less than $10 \%$ of cases, but in at least two series, that of Upmark (1930) and Passarge (1967), as many as $25 \%$ had other abnormalities. A wide variety of these have been recorded but Down's syndrome seems to occur considerably more frequently than one might expect by chance (Passarge, 1967). Pyloric stenosis and anal atresia also occur rather more often. In only one instance, case 77 of Upmark, was there any abnormality of the digits (such as syndactyly), and there appears to be no previous record of Hirschsprung's disease being associated with the distinctive skeletal malformations seen in our case in addition to congenital heart disease and phimosis.

There is unequivocal evidence for the familial occurrence of Hirschsprung's disease (Althoff, 1962; Bodian and Carter, 1963; Madsen, 1964; Emanuel et al, 1965; Passarge, 1967). However, no simple hypothesis can be put forward for the genetic factors involved in the vast majority of cases. There is no evidence for autosomal recessive or sex-linked recessive inheritance, and it seems more likely that the condition may yet be another example of sexmodified, multifactorial inheritance, where in the case of short segment Hirschsprung's disease more than three times as many girls as boys are affected. In our particular family, however, it is quite possible that one may be dealing with a unique recessively or X-linked disorder, as the two successive male sibs both had what appeared to be almost identical abnormalities.

Our experience of fetoscopy is similar to that of Scrimgeour (1974) and of Valenti (1973), in that the examination is best performed between 18 and 20 weeks' gestation and that prior placental localization is mandatory. The procedure should be performed at abdominal section, and a horizontal mattress or a purse-string suture should be around the proposed fetoscopy site. The uterine wall should be incised avoiding inadvertent rupture of the membranes. Fundal approach is preferred because it reduces the need for excessive manoeuvring. The point of entry into the amniotic cavity is furthermost from the cervix, thus perhaps reducing the later amniotic fluid loss and more important lessening the risk of ascending infection. In our case the source of the continual liquor loss was probably the defect in the membrane created at fetoscopy.

Fetoscopy as a form of investigation must be regarded as both risky and chancy. It is the former because of the high risk of precipitating a miscarriage, and of complications such as was experienced in our case. It is chancy because of the factors which may preclude visualization of the parts required to be seen for prenatal diagnosis, either because of the quality of the amniotic fluid, or the inability to manipulate the foetus or the instrument into a position where the relevant parts can be visualized. For these reasons fetoscopy ought to be reserved for serious, but not immediately lethal conditions which have distinct, easily recognizable external stigmata and for which there are no other more satisfactory methods of prenatal diagnosis. Therefore, it is probably best suited for conditions with abnormalities of the face particularly the lips, eyes, nose, and ears; the limbs; ventral surface of the abdominal wall and the external genitalia. The 
technique is probably unsuitable for the detection of a possible spina bifida, for we found that during the stage of pregnancy at which we carried out our examinations, in every case the fetus was presenting by the breech, facing the placenta, and with the back towards the uterine wall. Attempts to visualize the dorsal surface of the fetus have been unsuccessful, especially since manipulation of the fetus into different positions was not easily achieved.

We wish to thank our colleagues Dr W. E. Hunt for histological examination of the membranes, the Anaesthetic and Theatre Staff at the Royal Gwent Hospital for help, and Dr C. O. Carter for advice and critical comments. We also thank the Key-Med Company.

\section{REFERENCES}

Althoff, W. (1962). Zur Genetik der Hirschsprungischen Krankheit. Zeitschrift für menschliche Vererbungs-und Konstitutionslehre, 36, 314-340.
Bodian, M. and Carter, C. O. (1963). A family study of Hirschsprung's disease. Annals of Human Genetics, 26, 261-277.

Emanuel, B., Padorr, M. P., and Swenson, O. (1965). Familial absence of myenteric plexus. Fournal of Pediatrics, 67, 381-386.

Fraser, G. C. and Wilkinson, A. W. (1967). Neonatal Hirschsprung's disease. British Medical fournal, 2, 7-10.

Hofmann, S. and Rehbein, F. (1966). Hirschsprungsche Krankheit in Neugeborenalter. Zentralblatt für Kinderchirugie, 3, 182-194.

Laurence, K. M., Pearson, J. F., Prosser, R., Richards, C., and Rocker, I. (1974). Fetoscopy followed by live birth. (Letter.) Lancet, 2, 1120-1121.

Madsen, C. M. (1964). Hirschsprung's Disease. Munksgaard, Copenhagen and Charles C. Thomas, Springfield, Illinois.

Passarge, E. (1967). Genetics of Hirschsprung's disease. New England fournal of Medicine, 276, 138-143.

Scrimgeour, J. B. (1974). Fetoscopy. In Birth Defects, ed. by A. G. Motulsky and W. Lenz, pp. 234-239. Excerpta Medica, Amsterdam.

Upmark, E. Ask (1930). Studien über Megacolon. Beiträge zur klinischen Chirugie, 151, 267-317.

Valenti, C. (1973). Endoamniotic and Fetal Biopsy for Prenatal Genetic Diagnosis, pp. 26-27. International Congress Series, No. 297. Excerpta Medica, Amsterdam.

Wylie, G. G. (1957). Course and management of Hirschsprung's disease. Lancet, 1, 847-850. 